\title{
microRNA-182 inhibits the proliferation and invasion of human lung adenocarcinoma cells through its effect on human cortical actin-associated protein
}

\author{
LIFAN ZHANG ${ }^{1}$, TE LIU ${ }^{2,5}$, YONGYI HUANG ${ }^{3}$ and JUN LIU ${ }^{4}$ \\ ${ }^{1}$ Huadong Hospital Affiliated to Fudan University, Shanghai 200040; ${ }^{2}$ School of Environmental Science and Engineering, \\ Donghua University, Shanghai 201620, P.R. China; ${ }^{3}$ Laboratoire PROTEE, Bâtiment R, \\ Université du Sud Toulon-Var, 83957 La Garde Cedex, France; ${ }^{4}$ Waksman Institute, Rutgers, \\ The State University of New Jersey, New Brunswick, NJ 08854, USA
}

Received February 3, 2011; Accepted March 22, 2011

DOI: $10.3892 /$ ijmm.2011.679

\begin{abstract}
Lung cancer is one of the main causes of cancer death worldwide. The cortactin gene, CTTN, may play a pivotal role in the proliferation and invasion of tumors. A microRNA (miR-182) was cloned and used to study the expression of CTTN and its regulatory effects on the proliferation and invasion of the lung cancer cell line, A549. Cortactin protein and CTTN mRNA expression decreased in A549 cells that were transfected with the miR-182 expression plasmid. A cell proliferation assay indicated that miR-182 expression affected cell cycle regulation and suppressed proliferation of lung cancer cells in vitro. In addition, xenograft experiments confirmed the suppression of tumor growth in vivo, which was due to the promotion of apoptosis. In conclusion, endogenous mature miR-182 expression may have an important role in the pathogenesis of lung cancer through its interference with the target gene $C T T N$ by epigenetic modification.
\end{abstract}

\section{Introduction}

Lung cancer is the leading cause of cancer death worldwide. In 2008, it claimed the lives of approximately 1.4 million people around the world $(1,2)$ and its 5-year survival rate is $\sim 15 \%$ (3). In the past 20 years, lung cancer mortality has increased in China, especially in urban areas and among older people; treatment outcomes remain unsatisfactory $(4,5)$ and the mortality is high. Hence, there is great interest in studying

Correspondence to: Dr Te Liu, School of Environmental Science and Engineering, Donghua University, Shanghai 201620, P.R. China E-mail: liute79@yahoo.com

Present address: ${ }^{5}$ Shanghai Geriatric Institute of Chinese Medicine, Shanghai 200031, P.R. China

Key words: lung cancer, microRNA-182, cortical actin-associated protein, apoptosis, epigenetic this disease in order to better understand its biology and to translate research findings into improved therapies (6).

Since their first description in 1993 (7), microRNAs (miRNAs) have been shown to play key roles in developmental regulation, and in the maintenance of undifferentiated, or incompletely differentiated, cell types, such as stem cells $(8,9)$. The human genome is estimated to contain nearly 900 unique miRNAs, which control, in part, the expression of more than one third of the human genes (7). In human cancer, several dysregulated miRNAs have been shown to have oncogenic or tumor suppressive activity (4). For example, miRNA-155 (miR-155) is overexpressed in a variety of human cancers and immune diseases (10). Expression of BIC and miR-155 in three latency type III, EBV-positive Burkitt lymphoma (BL) cell lines, and in all cases of primary post-transplant lymphoproliferative disorder (PTLD) suggests a possible role for EBV latency type III-specific proteins in the induction of BIC expression $(11,12)$. In addition, miR-128 inhibits glioma cell proliferation by targeting the transcription factor, E2F3a (13), and miR-200c and miR-141 (members of the miR-200 family) are important regulators of the epithelial to mesenchymal transition in normal and cancer cells $(7,14)$. Furthermore, fragments of miRNA can be found and measured in the serum of patients with cancer, and could potentially act as a novel class of biomarkers for diagnosis or cancer staging; for instance, tumor-suppressive activities in human hepatocellular carcinoma cells are decreased by up-regulation of miR23a 27a 24 (15). However, the complexity of the structures and cloning processes for miRNAs has prevented the analysis of large numbers of clinical samples.

Cortical actin-associated protein (cortactin), an 80 and $85 \mathrm{kDa}$ protein, is a widely expressed actin-binding protein, which contains 6.537-amino acid repeats, of which the fourth is required for filamentous actin-binding activity (16-18). It was first identified as one of the major substrates for Src kinase (19) and has four major domains of interest, the $\mathrm{N}$-terminal acidic and tandem repeat domains, and the $\mathrm{C}$-terminal proline-rich and SH3 domains (19). The CTTN gene, also called EMS1, which codes the cortactin protein, is frequently overexpressed in breast, and head and neck cancers due to its presence in 
the 11q13 amplicon (19). This amplification has been associated with higher pathological stage, lymph node and distant metastases, and decreased survival. In addition, cortactin can stimulate the actin-nucleation activity of the Arp2/3 complex, alone or in combination with N-WASP (19-21). It also inhibits debranching and disassembly of dendritic actin networks.

Cellular roles for cortactin include the bridging of the endocytic machinery with components and regulators of the actin cytoskeleton, and the binding of the mechanochemical GTPase dynamin 2, which regulates the fission of endocytic vesicles. The function of cortactin has been examined in tumors grown in mice (19). Since cortactin overexpression increases the motility of fibroblasts and endothelial cells and increases bone metastasis in MDA-MB-231 breast cancer cells in a nude mouse model (20), one possible explanation for CTTN amplification in human cancers is that cortactin overexpression promotes tumor cell invasion and metastasis. The regulation of the endocytosis of integrins and growth factor receptors, or the secretion of molecules, such as proteases or extracellular matrix (ECM), by cortactin might have major effects on cell motility that would be cell context dependent (19). In vivo evidence supports a role for cortactin in metastasis, which is consistent with its well-characterized cell biological roles in cell motility and ECM degradation. Although known to be involved in cell motility and tumor occurrence, the mechanisms and characteristics of its regulation have not been addressed. Therefore, we sought to determine whether a miRNA existed that could specifically target cortactin to regulate its expression in different cells over time.

In this study, the miR-182 expression vector was transfected into the human lung adenocarcinoma cell line A549 to determine whether it could specifically regulate $C T T N$ and inhibit cell proliferation and invasion. If miR-182 expression were involved in this cell line, it could potentially be an important therapeutic target in human lung adenocarcinoma.

\section{Materials and methods}

Cell lines, animals. The human lung adenocarcinoma cell line A549 was grown in DMEM (Hyclone, Logan, USA) supplemented with $10 \%$ fetal bovine serum (FBS) (PAA Lab. Inc., Queensland, Australian), penicillin (100 U/ml), streptomycin (100 U/ml), and $2 \mathrm{mM} \mathrm{L-glutamine} \mathrm{(all} \mathrm{from} \mathrm{Hyclone).} \mathrm{A549}$ cells were maintained at $37^{\circ} \mathrm{C}$ in a humidified atmosphere of air containing $5 \% \mathrm{CO}_{2}$.

BALB/c nude mice were of 6-7 weeks of age and 20-22 g of weight. All animal procedures were carried out at Tongji University with the approval of the Institutional Animal Care and Use Committee and in accordance with institutional guidelines.

Vector construction. For expression experiments, plasmid pRNAT-CMV32-cGFP-mir182 (pre-miRNA of miRNA-182 expression element), oligonucleotide pairs for pre-miRNA of miRNA-182 and linker sequences with BamHI and XhoI sites were chemically synthesized (10). The sequences of the oligonucleotides were: top strand, 5'-GTggatccCTGTTTGGCAA TGGTAGAACTCACACTTTTTGCCTCCAACTGACT CCTACATATTAGCATTAACAGctcgagCC-3', and bottom strand, 5'-GGctcgagCTGTTAATGCTAATATGTAGGAGTC
AGTTGGAGGCAAAAAAGTGTGAGTTCTACCATTGC CAAACAGggatccAC-3' (sequences corresponding to miRNA182 seed sequences are capitalized, underlined and bold; restriction enzyme sites are in lower case and underlined). To build the expression plasmid the pairs of oligos were annealed and inserted into the multiple cloning sites between the BamHI and XhoI sites in the pRNAT-CMV32-cGFP/Neo vector (GenScript, New Jersey, USA). The negative control plasmid pRNAT-CMV32-cGFP-mir182-Mut was similarly built, except that 17 nucleotides in sequences corresponding to the miRNA-182 seed sequences were mutated (change from TTTGGCAATGGTAGAAC to TaTGcgAtTGGaAcAtg, mutations shown in lower-case). Co-transfection of the A549 cell line was conducted to transfer $0.3 \mu \mathrm{g}$ miRNA-182 expressed vector or miRNA-182 mutant vector, respectively, with Lipofectamine 2000 reagent according to the manufacturer's protocol.

$R N A$ extraction and analysis by quantitative real-time PCR $(q R T-P C R)$. Total-RNA from each group of cells was isolated using TRIzol reagent (Invitrogen) according to the manufacturer's protocol. The RNA samples were treated with DNase I (Sigma-Aldrich, St. Louis, MO, USA), quantified, and reversetranscribed into cDNA using the ReverTra Ace- $\alpha$ first strand cDNA synthesis kit (Toyobo). qRT-PCR was conducted using a RealPlex4 real-time PCR detection system from Eppendorf Co. Ltd. (Germany), with SYBR-Green real-time PCR Master mix (Toyobo) used as the detection dye. qRT-PCR amplification was performed over 40 cycles with denaturation at $95^{\circ} \mathrm{C}$ for $15 \mathrm{sec}$ and annealing at $58^{\circ} \mathrm{C}$ for $45 \mathrm{sec}$. Target cDNA was quantified using the relative quantification method. A comparative threshold cycle $(\mathrm{Ct})$ was used to determine gene expression relative to a control (calibrator) and steady-state mRNA levels are reported as an $n$-fold difference relative to the calibrator. For each sample, the maker genes $\mathrm{Ct}$ values were normalized using the formula $\Delta \mathrm{Ct}=\mathrm{Ct} \_$genes $-\mathrm{Ct} \_$18sRNA. To determine relative expression levels, the following formula was used $\Delta \Delta \mathrm{Ct}=\Delta \mathrm{Ct} \_$all_groups $-\Delta \mathrm{Ct} \_b l a n k c o n t r o l \_g r o u p$. The values used to plot relative expressions of markers were calculated using the expression $2^{-\Delta \Delta \mathrm{Ct}}$. The mRNA levels were calibrated based on levels of 18S rRNA. The cDNA of each gene was amplified as previously described using the following primers: CTTN-forward: 5'-TAGAGCCTGGTGCCTGGG-3', CTTN-reverse: 5'-TAATCCAATGAGGAATTTCCAG-3'.

Methyl thiazolyl tetrazolium (MTT) assay for cell proliferation. Each group of A549 cell lines was seeded at $2 \times 10^{3} /$ well in 96-well plates and cultured in DMEM supplemented with $10 \% \mathrm{FBS}$ at $37^{\circ} \mathrm{C}$ with $5 \% \mathrm{CO}_{2}$, until $85 \%$ confluent. MTT (Sigma-Aldrich) reagent $(5 \mathrm{mg} / \mathrm{ml})$ was added to the maintenance cell medium at different time points, and incubated at $37^{\circ} \mathrm{C}$ for an additional $4 \mathrm{~h}$. The reaction was terminated with $150 \mu 1$ dimethylsulfoxide (DMSO, Sigma-Aldrich) per well. The cells were lysed for $15 \mathrm{~min}$ and the plates were gently shaken for $5 \mathrm{~min}$. Absorbance values were determined by using the enzyme-linked immunosorbent assay (ELISA) reader (Model 680, Bio-Rad) at $490 \mathrm{~nm}$.

Flow cytometric (FCM) analysis of cell cycle by propidium iodide (PI) staining. Each group of A549 cells was seeded at 
$3 \times 10^{5} /$ well in 6 -well plates and cultured until $85 \%$ confluent. Each group of cells was washed with PBS three times, and the cells were then collected by centrifugation (Allegra X-22R, Beckman Coulter) at $1000 \mathrm{x}$ g for $5 \mathrm{~min}$. The cell pellets were resuspended in $1 \mathrm{ml}$ of PBS, fixed in 70\% ice-cold ethanol, and kept in a freezer for more than $48 \mathrm{~h}$. Before flow cytometric analysis, The fixed cells were centrifuged, washed twice with PBS, and resuspended in PI staining solution (Sigma-Aldrich) containing $50 \mu \mathrm{l} / \mathrm{ml} \mathrm{PI}$ and $250 \mu \mathrm{g} / \mathrm{ml}$ RNase A (SigmaAldrich). The cell suspension, which was hidden from light, was incubated for $30 \mathrm{~min}$ at $4^{\circ} \mathrm{C}$ and analyzed using FACS (FCM-500, Beckman Coulter). A total of 20,000 events were acquired for the analysis using the CellQuest software.

Luciferase reporter assay. All steps of the luciferase reporter assay were performed as previously described $(15,22)$. NIH-3T3 cells were seeded at $3 \times 10^{4}$ cells/well in 48 -well plates and co-transfected with $400 \mathrm{ng}$ of pRNAT-CMV32-cGFP-mir182 or pRNAT-CMV32-cGFP or pRNAT-CMV32-cGFP-mir182-Mut, $20 \mathrm{ng}$ of pGL3cm-CTTN-3UTR-WT or pGL3cm-CTTN3UTR-Mut, and pRL-TK (Promega, Madison, USA) using the Lipofectamine 2000 reagent according to the manufacturer's protocol. After $48 \mathrm{~h}$ of transfection, the luciferase activity was measured using the dual-luciferase reporter assay system (Promega).

Wound-healing assay. All steps of the assay were conducted according to a previously described protocol (17). A549 cells were grown to confluence and then wounded using a $200 \mu \mathrm{l}$ pipette tip. Three wounds were made for each sample, and all were photographed at the zero time point and at subsequent time points. Assays were repeated thrice in each clone.

Northern blotting. All steps of Northern blotting were performed as previously described (23). For all groups, $20 \mu \mathrm{g}$ of good quality total RNA were analyzed on a $7.5 \mathrm{M}$ urea, $12 \%$ PAA denaturing gel and transferred to a Hybond- $\mathrm{N}^{+}$ nylon membrane (Amersham, Freiburg, Germany). Membranes were cross-linked using UV light for $30 \mathrm{sec}$ at $1200 \mathrm{~mJ} / \mathrm{cm}^{2}$. Hybridization was performed with the miRNA-182 antisense StarFire probe, 5'-TTTGGCAATGGTAGAACTCACACT-3' (IDT, Coralville, IA), to detect the 22-nt miRNA-182 fragments according to the instruction of the manufacturer. After washing, membranes were exposed for 20-40 h to Kodak XAR-5 films (Sigma-Aldrich). As a positive control, all membranes were hybridized with a human U6 snRNA probe, 5'-GCAGGGGCCATGCTAATCTTCTCTGTATCG-3'. Exposure times for the U6 control probe varied from 15-30 min.

Immunofluorescence staining analysis for relative protein expression. The cultured cells were washed 3 times with PBS and fixed with $4 \%$ paraformaldehyde (Sigma-Aldrich) for $30 \mathrm{~min}$. After blocking, the cells were incubated with a rabbit anti-human CTTN polyclonal antibody (1:200; Santa Cruz Biotechnology, Sana Cruz, CA) overnight at $4^{\circ} \mathrm{C}$, and then with a Cy3-conjugated goat anti-rabbit IgG antibody (1:200; Abcam, Cambridge, UK) and $5 \mathrm{mg} / \mathrm{ml}$ DAPI (Sigma-Aldrich) at room temperature for $30 \mathrm{~min}$. The cells were then thoroughly washed with TBST and viewed under a fluorescence microscope (DMI3000; Leica, Allendale, NJ, USA).
Western blotting. Total protein extracts of each group of cells were resolved by $12 \%$ SDS-PAGE and transferred on PVDF (Millipore) membranes. After blocking, the PVDF membranes were washed 4 times for 15 min with TBST at room temperature and incubated with the primary antibody (rabbit anti-human CTTN polyclonal antibody (1:500). Following extensive washing, membranes were incubated with the secondary peroxidase-linked goat anti-rabbit IgG (1:1000, Santa Cruz Biotechnology) for $1 \mathrm{~h}$. After washing 4 times for 15 min with TBST at room temperature once more, the immunoreactivity was visualized using the enhanced chemiluminescence ECL kit (Pierce Biotechnology), and the membranes were exposed to Kodak XAR-5 film.

In vivo xenograft experiments. About $1 \times 10^{6}$ logarithmically growing A549 cells were inoculated in BALB/c nude/nude mice. Each experimental group consisted of four mice. After 4 weeks of observation, the mice were sacrificed and tumors were stripped (24). Tumor weight was measured and tumor volume was calculated according to the formula: Tumor volume $\left(\mathrm{mm}^{3}\right)=$ length $(\mathrm{mm}) \mathrm{x}$ width $(\mathrm{mm}) \mathrm{x}$ height $(\mathrm{mm})$.

Statistical analysis. Each experiment was performed at least three times, and the data are shown as the mean \pm SE where applicable. Differences were evaluated using Student's t-tests. The probability of $<0.05$ was considered to be statistically significant.

\section{Results}

Bioinformatic analysis of miR-182 and the 3'-untranslated region of CTTN $m R N A$. The precursor miRNA (pre-miRNA) sequences, mature miRNA sequences, chromosomal locations, and length of miR-182 and the target gene CTTN were analyzed in multiple species using the online research tool, the miRBase Target database (http://www.mirbase.org) $(23,25)$. Seven putative miRNA target sites were identified in the 3'-untranslated region (3'-UTR) of CTTN mRNA, depending on the species. For this study, we focused on the human miR-182, which may target the human CTTN 3'-UTR, because these sites are conserved, to varying degrees, across species (Fig. 1).

Identification of miR-182 binding sites in the CTTN 3'-UTR region. Plasmid DNA of each CTTN mRNA 3'-UTR site [wild-type (wt) CTTN, empty plasmid, and mutant CTTN] was co-transfected with the miR-182 expression vector (wt miR-182, empty vector, and mutant miR-182) into the mouse embryonic fibroblast cell line, NIH-3T3, to examine whether CTTN gene expression was regulated by the mature miR-182. Luciferase activity of the CTTN 3'-UTR sites was significantly inhibited by wt miR-182 (Fig. 1), while luciferase activity of the mutated CTTN 3'-UTR sites was not inhibited, which indicate that miR-182 targets CTTN.

miR-182 specifically influences the expression of cortactin protein in the lung cancer cell line. Northern blot analysis showed that the hybridized signal of mutant miR-182 in the lung cancer cell line, A549, was weaker than in one of the miR-182 transfected. Immunofluorescence (IF) staining, 
A

H. Spaiens UUUGGCARGGUAGMCUCACACU--

M. Musculus UUUGGCA.sUGGU.G..ACUCACACCG-

B. Rerio --UGG---UUCUAG মCUUGCCAACUA.

B

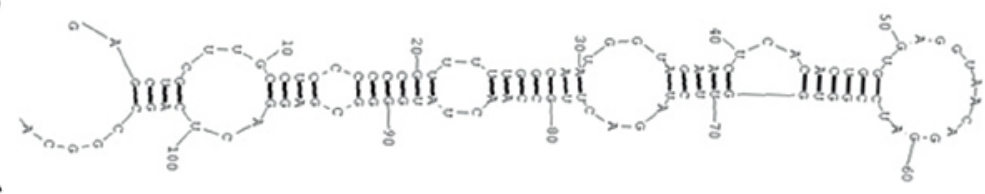

C

Human CTTN mRNA 3' UTR (974-980bp)

Human mature miR-182

5. ...g6gggugUaguaUUUUUGC.

3. UchcacucabaugguahcguU 5 .

D

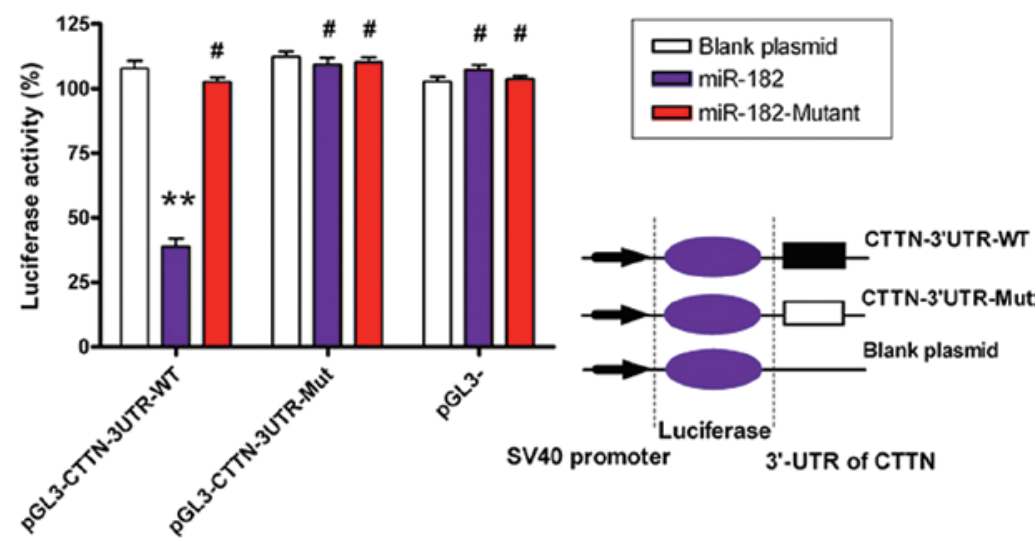

Figure 1. The human CTTN microRNA (miRNA) 3'-untranslated region (3'-UTR) contains microRNA-182 (miR-182) binding sites. The expression of miR182 and its interference with the target gene CTTN was assessed by a luciferase assay. (A) The mature miR-182 sequences of multiple species analyzed and contrasted by bioinformatic tools. (B) The typical secondary structure of the precursor miRNAs (pre-miRNAs) with miR-182. There are stem-loop and hairpin structures in the pre-miRNA, and the common binding site is located in an unstable region with a multi-branching loop-like RNA structure. The mature miRNAs are bound in the 3'-UTR of the target gene. (C) Complementarity between miR-182 and the putative human CTTN 3'-UTR site target (974-980 bp downstream) showing that the conserved bases of the putative miR-182 target sequence are present in the human CTTN 3'-UTR. (D) Wild-type (wt) reporter or mutated control luciferase plasmids were transfected into NIH-3T3 cells with miR-182 expression plasmids or mutant-miR-182 expression plasmids. Luciferase activity within the CTTN 3'-UTR sites is inhibited by miR-182 ( ${ }^{* *} \mathrm{P}<0.01$ and ${ }^{*} \mathrm{P}>0.05$ vs. the blank plasmid; $\left.\mathrm{n}=3\right)$.

quantitative real-time PCR (qRT-PCR) and Western blotting were used to determine whether exogenous and endogenous miR-182 could influence the expression of cortactin. The IF assay revealed that cortactin protein expression decreased in A549 cells that were transfected with the miR-182 expression plasmid compared to miR-182 mutant transfected and to untransfected cells (Figs. 2 and 3). Therefore, miRNA-182 expression may influence endogenous cortactin expression.

The qRT-PCR results confirmed the IF results: when the miR-182 expression vector was transfected into the A549 cells, the CTTN mRNA expression decreased compared to that of untransfected and mutant-miR-182 transfected cells. The mRNA expression was normalized to that of the $18 \mathrm{~S}$ ribosomal RNA (rRNA), which served as an internal control (Fig. 2). Interestingly, Western blotting revealed that cortactin levels in untransfected cells or mutant-miR-182 transfected cells were $0.892 \pm 0.107$ and $0.795 \pm 0.137$ of the GAPDH levels, respectively (Fig. 2). These values were significantly higher than those for the miR-182 transfected group $(0.289 \pm 0.179$ of GAPDH), which indicated that exogenous miR-182 downregulated cortactin expression.
Cell cycle changes induced by miR-182. To detect changes in cell cycle progression, miR-182 transfected, mutant-miR-182 transfected, and untransfected A549 cells were stained with PI and analyzed by flow cytometry. As shown in Fig. 3, most of the miR-182 transfected cells were arrested in the $G_{0} / G_{1}$ phase of the cell cycle and the percentage of cells in the $S$ and $\mathrm{G}_{2} / \mathrm{M}$ phases were markedly decreased (Table I). In contrast, there were no significant differences in the cell cycle distribution of mutant-miR-182 transfected and untransfected cells. These cell cycle results suggest that miR-182 expression affects cell cycle regulation in lung cancer cells in vitro.

Proliferation of A549 cells is inhibited by miR-182. The results of the proliferation assays performed are shown in Fig. 2. Using an MTT assay, the survival rate of miR-182 transfected cells at both $72 \mathrm{~h}$ and $96 \mathrm{~h}$ post-transfection was shown to be markedly lower than untransfected cells and mutant-miR-182 transfected cells. In contrast, the viability of untransfected cells, mutant-miR-182 transfected cells, and miR-182 transfected cells showed no differences at $24 \mathrm{~h}$. Survival rates remained unchanged for untransfected and 
A

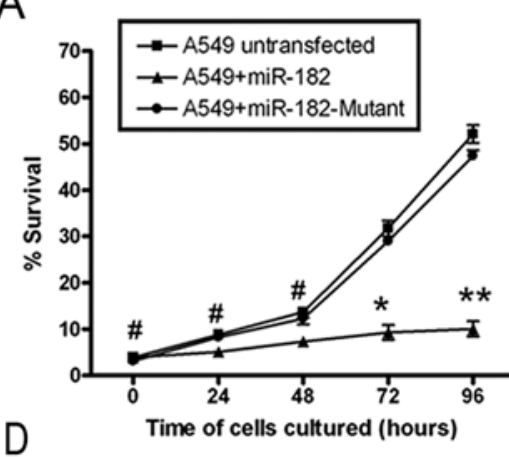

D

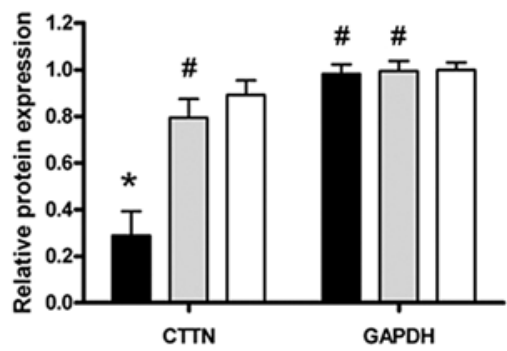

B

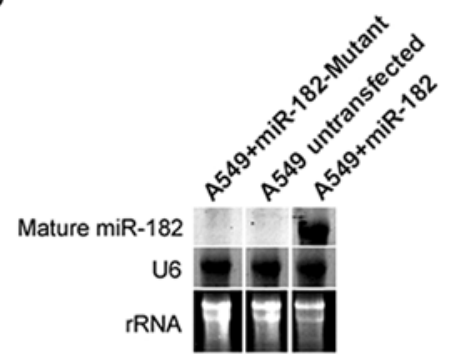

C

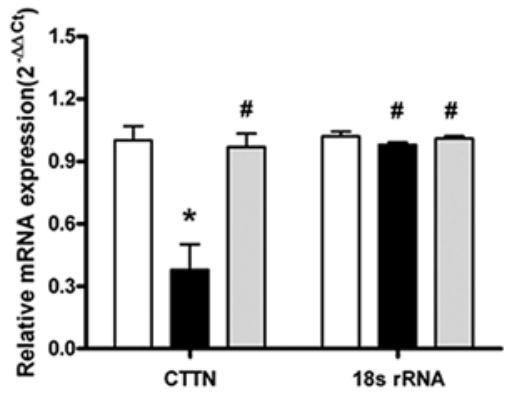

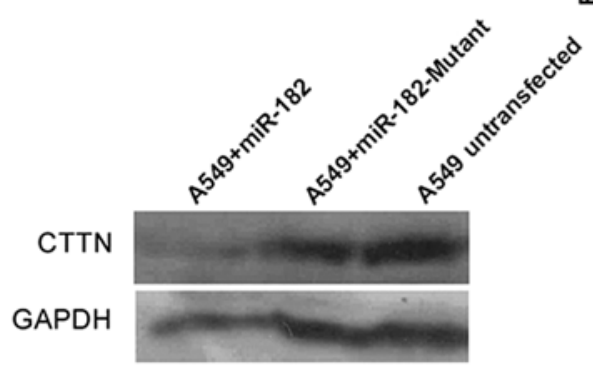

Figure 2. miR-182 and CTTN expression in different groups and the effect of miR-182 on cell proliferation. (A) MTT assay showing that the survival rate of miR-182 transfected cells is markedly lower than untransfected and mutant-miR-182 transfected cells at both $72 \mathrm{~h}$ and $96 \mathrm{~h}$ post-transfection $(* \mathrm{P}<0.05$, ${ }^{* *} \mathrm{P}<0.01$ and ${ }^{\#} \mathrm{P}>0.05$ vs. the blank plasmid; $\mathrm{n}=3$ ). (B) Northern blot analysis showing that the hybridized signal of mutant miR-182 in the A549 cell line is weaker than that of miR-182 transfected. The human U6 probe was used as a loading control. (C) qRT-PCR indicating that the CTTN mRNA expressed in the miR-182 transfected group is lower than in the untransfected or mutant-miR-182 transfected group. Relative mRNA expression is shown after normalization to 18S rRNA, which served as an internal control. (D) Western blot analysis showing the expression of CTTN. CTTN levels in untransfected cells or mutantmiR-182 transfected cells are significantly higher than those of the miR-182 transfected group. The data indicate that exogenous miR-182 down-regulates CTTN expression ( $\mathrm{P}<0.05$ and ${ }^{\#} \mathrm{P}>0.05$ vs. the untransfected group; $\mathrm{n}=3$ ).

Table I. Cell cycle changes of A549 cells induced by miR-182.

\begin{tabular}{lcrr}
\hline & $\mathrm{G}_{0} / \mathrm{G}_{1}$ phase $(\%)$ & S phase $(\%)$ & $\mathrm{G}_{2} / \mathrm{M}_{\text {phase }(\%)}$ \\
\hline miR-182 & $84.61 \pm 1.23$ & $7.83 \pm 1.09$ & $7.56 \pm 1.44$ \\
Untransfected & $64.31 \pm 1.16$ & $15.30 \pm 1.27$ & $20.39 \pm 1.31$ \\
Mutant-miR-182 & $60.19 \pm 2.01$ & $17.58 \pm 0.94$ & $22.23 \pm 1.76$ \\
\hline
\end{tabular}

mutant-miR-182 transfected cells for the remainder of the time course, which indicated that induced exogenous miR-182 expression inhibited the growth of lung cancer A549 cells in vitro.

Wound-healing assays also showed that the miR-182 transfected cells migrated more slowly than the mutant-miR-182 transfected cells and untransfected cells. Both the mutantmiR-182 transfected and the untransfected cells had become confluent at $24 \mathrm{~h}$ post wounding, but the miR-182 transfected cells had migrated only a 2- to 4-cell distance (Fig. 3).

Expressed miR-182 in A549 cells inhibits subcutaneous tumor growth in nude mice. The effect of miR-182 expression on tumor growth was investigated in vivo by subcutaneous inoculation of the miR-182 transfected A549 cell line or the untransfected cell line into two groups of nude mice. All mice in the untransfected group developed tumors approximately 28 days after injection, whereas, only 1 in 4 mice from the miR-182 transfected group developed tumor by this time. Although both groups developed tumors, the tumors formed by miR-182 transfected cells grew more slowly than those in the untransfected group (Fig. 4). In addition, when the mice were sacrificed 56 days after injection, the tumor weights of the untransfected group were significantly heavier than those of the miR-182 transfected group. Furthermore, miR-182 expression in the A549 cell lines led to a significant decrease in the tumor volume (Fig. 4).

Ki-67 (a cell proliferation-related protein) was analyzed on the tumor sections by immunohistochemistry because tumor growth is determined by the balance of cell proliferation and programmed cell death. The tumors formed by untransfected A549 cells showed positive or strongly positive staining compared to the controls, but the tumors formed by miR-182 transfected cells exhibited only weak immunoreactions (Fig. 4). Kaplan-Meier survival curves for the mice inoculated with miR-182 transfected cells and untransfected cells were significantly different. In contrast, more apoptotic cells were detected in tumors from the miR-182 transfected cells. These results indicate that miR-182 expression in lung cancer cells suppresses in vivo tumor growth by promoting apoptosis. 


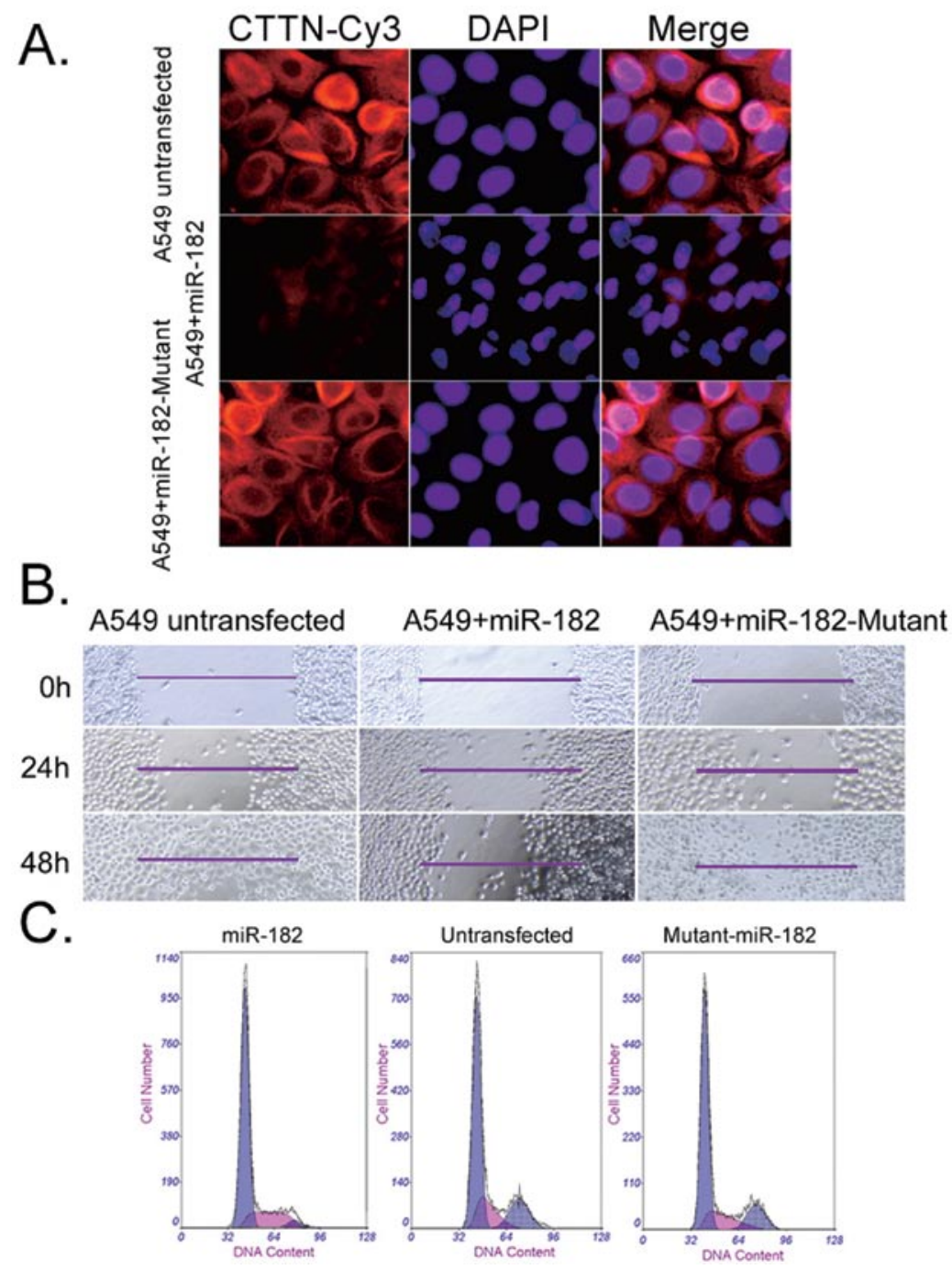

Figure 3. Exogenous miR-182 can not only influence the cortactin expression, but also the proliferation and cell cycle of the A549 cell line. (A) Immunofluorescence (IF) staining showing that exogenous miR-182 influences the expression of cortactin. Cortactin protein expression is decreased in the A549 cell line transfected with the miR-182 expression plasmid, compared with those cells transfected with the mutant-miR-182 expression plasmid or with untransfected cells. (B) Wound-healing assay showing that exogenous miR-182 can influence proliferation of A549 cells. The wound-healing assay showing that the miR-182 transfected A549 cells migrate more slowly than the mutant-miR-182 transfected and the untransfected cells. (C) Flow cytometry showing that exogenous miR-182 can influence the cell cycle of the A549 cell line. It shows that most of the miR-182 transfected cells were arrested in the $\mathrm{G}_{0} / \mathrm{G}_{1}$ phase of the cell cycle and the percentage of cells in the $\mathrm{S}$ and $\mathrm{G}_{2} / \mathrm{M}$ phases were markedly decreased.

\section{Discussion}

There is increasing evidence supporting that cortactin is a prominent substrate of Src-protein-tyrosine kinase $(18,26)$, and that tyrosine phosphorylation of cortactin occurs frequently in response to various extracellular stimuli, including growth factors, bacterially-mediated phagocytosis, cell shrinkage and membrane injury. Although studies have found that the introduction of a mutant cortactin deficient in tyrosine phosphorylation impairs cell motility, tumor invasion, invadopodia, and podosome formation $(18,20,27,28)$, and that cortactin plays important roles in tumor growth, there have been few reports on the role of cortactin regulation in tumors by epigenetic modification. Studies have however, increasingly shown that miRNAs play important roles in tumor occurrence, proliferation and invasion by interference with the expression of certain target cancer inhibitor genes. In addition, Sun et al indicated that the expression of endogenous RGS17 factor is regulated by miR-182 in human lung cancer cell lines; furthermore, ectopic expression of miR-182 significantly inhibits lung cancer cell proliferation and anchorage-independent cell growth (3). When bioinformatic tools were used to search for candidate genes related to miR-182, it was surprisingly found that miR-182 could putatively interfere with the expression of CTTN through sites located in the CTTN 3'-UTR region. We therefore, hypothesized that miR-182 may suppress human lung cancer A549 cells through down-regulation of CTTN expression.

Putative miRNA target sites in the 3'-UTR of CTTN mRNA were used to construct a miR-182 expression vector, which was then transfected into the human lung cancer cell line, A549. The luciferase activity assay indicated that the activity of the CTTN 3'-UTR site was significantly inhibited by miR-182, while that of the mutated CTTN 3'-UTR site was unchanged, which suggests that miR-182 targets CTTN. IF staining, qRT-PCR and Western blotting showed that cortactin protein expression decreased in the A549 cell line that was 

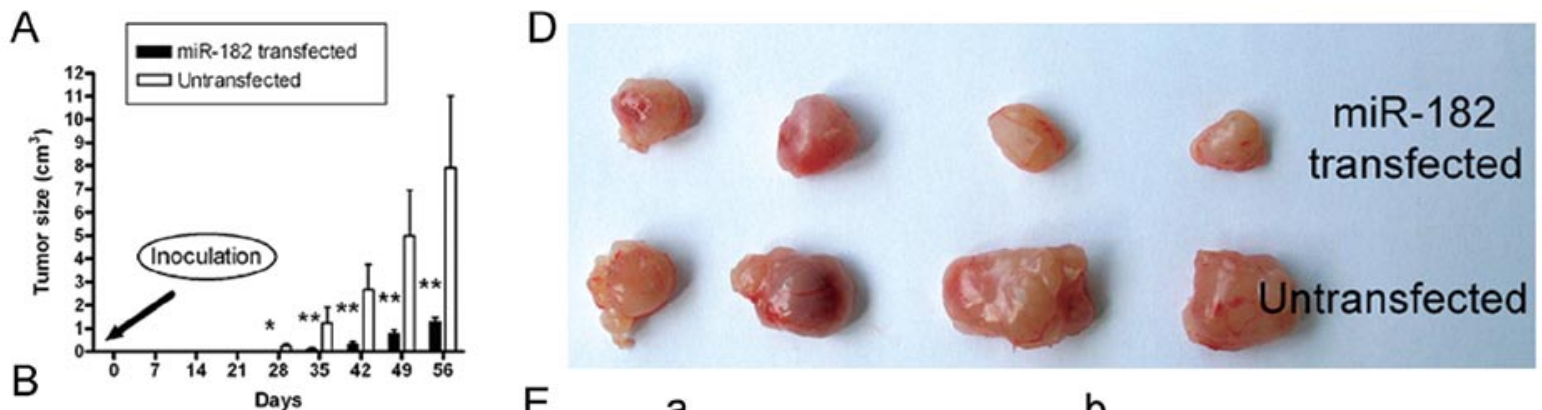

E

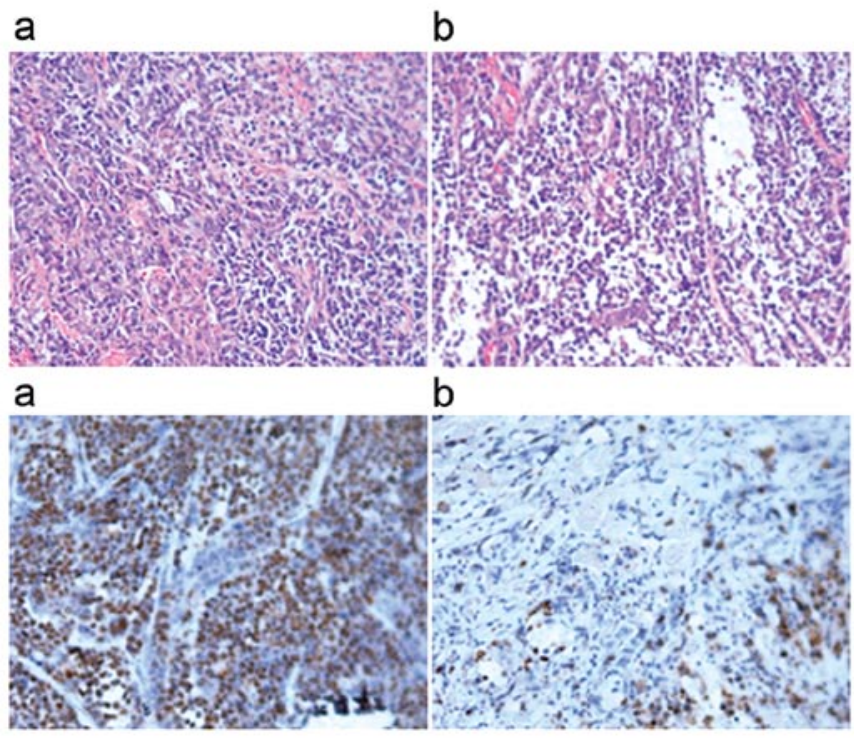

Figure 4. The results of xenograft experiments. (A) All mice of the untransfected group developed tumors approximately 28 days after injection. The tumors formed by the miR-182 transfected cells grew more slowly ( $\mathrm{P}<0.05$ and ${ }^{* * *} \mathrm{P}<0.01$ vs. untransfected cells; $\left.n=4\right)$. (B) The tumor weight of the untransfected group is heavier than those of the miR-182 transfected group, and miR-182 expression leads to a decrease in the tumor volume ("P<0.01 vs. untransfected; $n=4)$. (C) Kaplan-Meier survival curves of nude mice inoculated with A549 cell lines showing marked differences between the miR-182 transfected and untransfected groups. (D) Macroscopic appearance of the excised tumors in the transfected miR-182 and untransfected groups. (E) Hematoxylin and eosin staining reveals the cellular heterogeneity in pathological sections of the excised tumor tissue (a) untransfected group; (b) miR-182 transfected group (original magnification, x100). (F) Immunohistochemistry shows that the tumors formed by (a) untransfected A549 cells are positive or strongly positive with Ki-67 stain; whereas (b) the tumors formed by miR-182 transfected cells are weakly positive (original magnification, x100).

transfected with the miR-182 expression plasmid, compared with those transfected with the mutant miR-182 expression plasmid or untransfected cells. Flow cytometry revealed that most of the miR-182 transfected cells were arrested in the $\mathrm{G}_{0} / \mathrm{G}_{1}$ phase of the cell cycle with reduced percentages in the $\mathrm{S}$ and $\mathrm{G}_{2} / \mathrm{M}$ phases, which suggested that miR-182 expression could affect cell cycle regulation of lung cancer cells in vitro. Similarly, the MTT assay indicated that exogenous miR-182 expression inhibited the growth of the A549 cell line in vitro and the wound-healing assay showed that the miR-182 transfected cells migrated more slowly. Lastly, the results of xenograft experiments indicated that miR-182 expressed in the A549 cell line also suppressed tumor growth in vivo by promotion of apoptosis.

In contrast to a previous report that exogenous miR-182 expression in human lung cancer cell lines inhibited endogenous RGS17 protein expression, in this study it was found that CTTN expression was decreased in the miR-182 transfected cell line. The results not only suggest that the expression of cortactin was increased in the cell line, but also that miR-182 expression could influence endogenous CTTN expression. Taken together, these results suggest that the proliferation and invasion of human lung cancer cells may be related to
miR-182 expression. Inhibition or loss of miR-182 expression may result in excessive cortactin expression, and thus, influence tumor growth. Further studies will be required to fully clarify the function of miR-182, but it may potentially play an important role in human lung cancer.

\section{Acknowledgements}

This study was supported by grants from the Shanghai Committee Medical Science Foundation of China (no. 10411967100), the Medicine-Engineering Unite Fund for Shanghai Jiaotong University (no. YG2009MS47) and the Natural Science Foundation of the School of Medicine of Shanghai Jiaotong University (YZ1046).

\section{References}

1. Meng J, Xie W, Cao L, Hu C and Zhe Z: shRNA targeting HDGF suppressed cell growth and invasion of squamous cell lung cancer. Acta Biochim Biophys Sin (Shanghai) 42: 52-57, 2010.

2. Doebele RC, Oton AB, Peled N, Camidge DR and Bunn PA Jr: New strategies to overcome limitations of reversible EGFR tyrosine kinase inhibitor therapy in non-small cell lung cancer. Lung Cancer 69: 1-12, 2010. 
3. Sun Y, Fang R, Li C, et al: Hsa-mir-182 suppresses lung tumorigenesis through down-regulation of RGS17 expression in vitro. Biochem Biophys Res Commun 396: 501-507, 2010.

4. Guo X, Chen Y, Xu Z, Qian Y and Yu X: Prognostic significance of VEGF-C expression in correlation with COX-2, lymphatic microvessel density, and clinicopathologic characteristics in human non-small cell lung cancer. Acta Biochim Biophys Sin (Shanghai) 41: 217-222, 2009.

5. Zhang X, Zhang Z, Chen G, et al: FK228 induces mitotic catastrophe in A549 cells by mistargeting chromosomal passenger complex localization through changing centromeric $\mathrm{H} 3 \mathrm{~K} 9$ hypoacetylation. Acta Biochim Biophys Sin (Shanghai) 42: 677-687, 2010

6. Gazdar AF, Gao B and Minna JD: Lung cancer cell lines: Useless artifacts or invaluable tools for medical science? Lung Cancer 68: 309-318, 2010.

7. Vrba L, Jensen TJ, Garbe JC, et al: Role for DNA methylation in the regulation of miR-200c and miR-141 expression in normal and cancer cells. PLoS One 5: e8697, 2010.

8. Lee RC,Feinbaum RL and Ambros V: The C.elegans heterochronic gene lin-4 encodes small RNAs with antisense complementarity to lin-14. Cell 75: 843-854, 1993.

9. Carrington JC and Ambros V: Role of microRNAs in plant and animal development. Science 301: 336-338, 2003.

10. Jiang S, Zhang HW, Lu MH, et al: MicroRNA-155 functions as an OncomiR in breast cancer by targeting the suppressor of cytokine signaling 1 gene. Cancer Res 70: 3119-3127, 2010.

11. Luzi E, Marini F, Sala SC, Tognarini I, Galli G and Brandi ML: Osteogenic differentiation of human adipose tissue-derived stem cells is modulated by the miR-26a targeting of the SMAD transcription factor. J Bone Miner Res 23: 287-295, 2008.

12. Eis PS, Tam W, Sun L, et al: Accumulation of miR-155 and BIC RNA in human B cell lymphomas. Proc Natl Acad Sci USA 102: 3627-3632, 2005.

13. Zhang Y, Chao T, Li R, et al: MicroRNA-128 inhibits glioma cells proliferation by targeting transcription factor E2F3a. J Mol Med 87: 43-51, 2009.

14. Sithanandam G, Fornwald LW, Fields J and Anderson LM Inactivation of ErbB3 by siRNA promotes apoptosis and attenuates growth and invasiveness of human lung adenocarcinoma cell line A549. Oncogene 24: 1847-1859, 2005.

15. Huang S, He X, Ding J, et al: Up-regulation of miR-23a approximately 27 a approximately 24 decreases transforming growth factor-beta-induced tumor-suppressive activities in human hepatocellular carcinoma cells. Int J Cancer 123: 972-978, 2008.
16. Gibcus JH, Mastik MF, Menkema L, et al: Cortactin expression predicts poor survival in laryngeal carcinoma. Br J Cancer 98: 950-955, 2008

17. Luo ML, Shen XM, Zhang Y, et al: Amplification and overexpression of CTTN (EMS1) contribute to the metastasis of esophageal squamous cell carcinoma by promoting cell migration and anoikis resistance. Cancer Res 66: 11690-11699, 2006.

18. Luo C, Pan H, Mines M, Watson K, Zhang J and Fan GH: CXCL12 induces tyrosine phosphorylation of cortactin, which plays a role in CXC chemokine receptor 4-mediated extracellular signal-regulated kinase activation and chemotaxis. J Biol Chem 281: 30081-30093, 2006.

19. Grossman D, Kim PJ, Schechner JS and Altieri DC: Inhibition of melanoma tumor growth in vivo by survivin targeting. Proc Natl Acad Sci USA 98: 635-640, 2001.

20. Timpson P, Lynch DK, Schramek D, Walker F and Daly RJ: Cortactin overexpression inhibits ligand-induced down-regulation of the epidermal growth factor receptor. Cancer Res 65: 3273-3280, 2005.

21. Weaver AM: Cortactin in tumor invasiveness. Cancer Lett 265 : 157-166, 2008

22. He M, Xu Z, Ding T, Kuang DM and Zheng L: MicroRNA-155 regulates inflammatory cytokine production in tumor-associated macrophages via targeting C/EBPbeta. Cell Mol Immunol 6: $343-352,2009$

23. Kluiver J, Haralambieva E, de Jong D, et al: Lack of BIC and microRNA miR-155 expression in primary cases of Burkitt lymphoma. Genes Chromosomes Cancer 45: 147-153, 2006.

24. Carro MS, Lim WK, Alvarez MJ, et al: The transcriptional network for mesenchymal transformation of brain tumours. Nature 463: 318-325, 2010.

25. Martin MM, Lee EJ, Buckenberger JA, Schmittgen TD and Elton TS: MicroRNA-155 regulates human angiotensin II type 1 receptor expression in fibroblasts. J Biol Chem 281: 18277-18284, 2006.

26. Zhu J, Yu D, Zeng XC, Zhou K and Zhan X: Receptor-mediated endocytosis involves tyrosine phosphorylation of cortactin. J Biol Chem 282: 16086-16094, 2007.

27. Luo ML and Wang MR: CTTN (EMS1): an oncogene contributing to the metastasis of esophageal squamous cell carcinoma. Cell Res 17: 298-300, 2007.

28. Patel AS, Schechter GL, Wasilenko WJ and Somers KD: Overexpression of EMS1/cortactin in NIH3T3 fibroblasts causes increased cell motility and invasion in vitro. Oncogene 16 3227-3232, 1998. 